Setting The cardiac catheterisation laboratory in a regional heart centre in the UK.

Definitions The clinical indication for FFR measurement was the presence of an intermediate coronary stenosis $(50 \%-75 \%$ of the reference vessel diameter) which resulted in diagnostic and treatment uncertainty. FFR measurement was used to provide functional information on lesion severity and an FFR $<0.80$ was taken to represent a flow-limiting stenosis.

Methods The study involved three accredited interventional cardiologists and a study coordinator. The cardiologists separately reviewed the coronary angiograms and together with the clinical history, made a decision for medical therapy, PCI, CABG/MDT, or deferred management. The FFR results were then disclosed and the initial management decision was reviewed in light of the FFR result and changed as appropriate

Results Of 1621 acute NSTEMI patients (January 2009-March 2010) in our hospital, 100 (6.2\%) had FFR recorded. The treatment decisions for each cardiologist were: medical therapy $7 \%, 10 \%, 1 \%$; PCI $64 \%$, $70 \%, 60 \%$; CABG/MDT $13 \%, 12 \%, 15 \%$; deferred management $16 \%$, $8 \%, 24 \%)$. The proportion of patients allocated to each treatment group differed between the 2 nd and 3rd Cardiologist $(p=0.02)$. Following FFR disclosure, each cardiologist changed his/her treatment decision in $58 \%, 50 \%$ and $62 \%$ of patients $(p<0.05)$. Of the new decisions made following FFR disclosure, the proportion of patients allocated to medical therapy increased by $26 \%, 19 \%$ and $29 \%$, whereas the proportion of patients allocated to deferred management or multivessel PCI decreased by $16 \%, 8 \%, 24 \%$ and by $5 \%, 7 \%$ and $5 \%$, respectively (all $p<0.05$ ). The number of patients in whom the treatment decisions made by each cardiologist independently conformed (and so represented a consensus in at least 2 of the 3 decisions) increased from $74 \%$ to $92 \%$ as a result of FFR disclosure $(p<0.001)$.

Conclusion In our hospital about 1 in 20 NSTEMI had a coronary pressure wire study because of diagnostic uncertainty based on coronary angiography alone. In NSTEMI patients selected for FFR measurement, the FFR resulted in a change in management in at least half of the patients. FFR use increased the proportion of patients in whom treatment decisions conformed suggesting FFR use may also help to reduce the variation in treatment decisions using angiography alone. These results support further studies of the clinical utility and prognostic implications of FFR measurement in patients with NSTEMI.

\section{COMPARISON OF 4-H HEART FATTY ACID BINDING PROTEIN WITH 12-H TROPONIN I TO ASSESS 6-MONTH RISK FOLLOWING PERCUTANEOUS CORONARY INTERVENTION IN ACUTE CORONARY SYNDROMES}

doi:10.1136/heartjnl-2011-300198.22

${ }^{1} \mathrm{I}$ R Pearson, ${ }^{1} \mathrm{U}$ M Sivananthan, ${ }^{1} \mathrm{~J}$ H Barth, ${ }^{2} \mathrm{C}$ P Gale, ${ }^{1} \mathrm{~A}$ S Hall. ${ }^{1}$ Leeds Teaching Hospitals, Leeds, UK; ${ }^{2}$ Division of Biostatistics, University of Leeds, Leeds, UK

Background It is known that PCI can cause myocardial injury leading to the release of cardiac biomarkers into the circulation (procedural $\mathrm{MI}$ ). This occurs in approximately one third of procedures and has been shown to impact negatively on prognosis. Monitoring for procedural MI, although not yet standard practice, is increasingly undertaken as a measure of quality control, and may be a factor when deciding time of discharge from hospital following the procedure. The use of $\mathrm{TnI}$ to screen for procedural MI requires a wait of 12 -h post procedure before the blood sample may be taken, and an impact on length of hospital stay is inevitable. Heart-type Fatty Acid Binding Protein (H-FABP) is a small protein released rapidly and in large quantities from the myocardium into the circulation, both during ischaemia and following necrosis. It allows detection of myocardial injury associated with PCI earlier than with TnI.

Hypothesis $\mathrm{H}-\mathrm{FABP}$ at $4 \mathrm{~h}$ provides equivalent prognostic information to $\mathrm{TnI}$ at $12 \mathrm{~h}$ following PCI-induced myocardial injury.
Methods We studied 94 patients with ACS admitted to a single UK Teaching Hospital for PCI. We used the Randox Cardiac-Array to measure H-FABP at 4 hrs after PCI and troponin I at $12 \mathrm{~h}$ after PCI. Comparison of specificity and sensitivity of each biomarker for adverse cardiac events was made. Endpoint assessment consisted of one of the following three events (i) PC-induced MI (ii) readmission with MI by 6 months (iii) death by 6 months.

Results The area under the receiver operator curve was 0.73 for $\mathrm{H}-\mathrm{FABP}$ measured at $4 \mathrm{~h}$ as compared to 0.72 for TnI measured as $12 \mathrm{~h}$. Conclusion Early assessment of PCI-induced myocardial injury using the Randox Cardiac-Array to measure H-FABP is as sensitive and specific for adverse prognosis as is TnI measurement taken at $12 \mathrm{~h}$ post PCI. This approach should help to expedite early, safe hospital discharge following PCI.

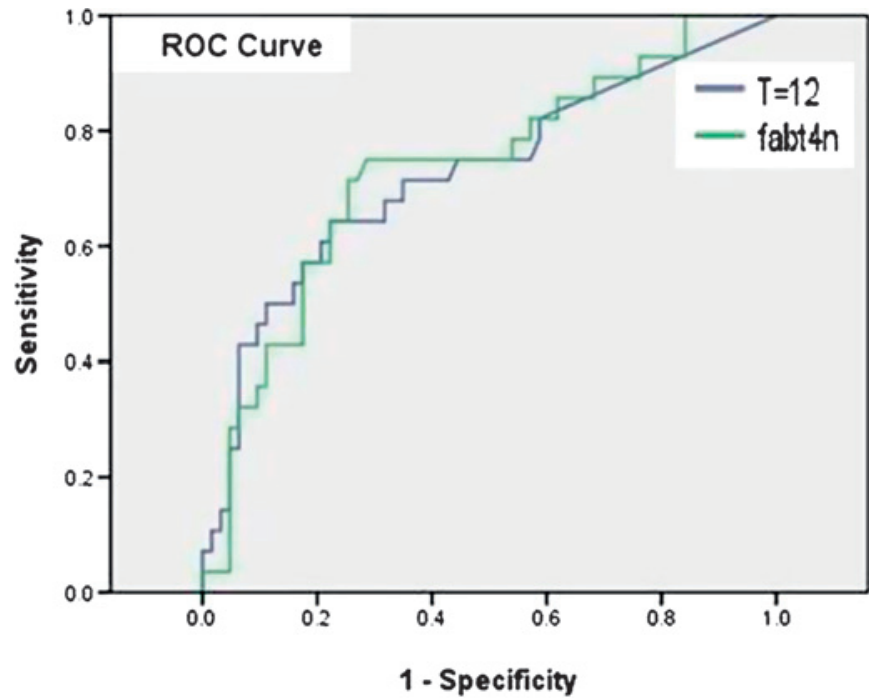

Abstract 22 Figure 1

23
SERUM NGAL IDENTIFIES CONTRAST NEPHROPATHY
EARLY IN PATIENTS WITH DIABETES MELLITUS AND
CHRONIC KIDNEY DISEASE UNDERGOING CORONARY
ANGIOGRAPHY AND ANGIOPLASTY

doi:10.1136/heartjnl-2011-300198.23

${ }^{1} \mathrm{~A}$ C Oureshi, ${ }^{2} \mathrm{R}$ Rampat, ${ }^{3} \mathrm{~S} \mathrm{M}$ Harwood, ${ }^{4} \mathrm{M}$ Roughton, ${ }^{2} \mathrm{M} \mathrm{M}$ Yaqoob, ${ }^{1} \mathrm{~A}$ Kapur. ${ }^{1}$ The London Chest Hospital, Barts and The London NHS Trust, London, UK; ${ }^{2}$ The Royal London Hospital, Barts and The London NHS Trust, London, UK; ${ }^{3}$ The William Harvey Research Institute, London, UK; ${ }^{4}$ The Royal College of Physicians, London, UK

Background The incidence of contrast nephropathy (CIN) following coronary angiography or percutaneous coronary intervention (PCI) in patients with diabetes mellitus (DM) may be up to $30 \%$ and is associated with increased long term morbidity and mortality.

Methods We recruited 208 consecutive patients undergoing elective or urgent coronary angiography or PCI with known DM and chronic kidney disease (CKD) (defined as eGFR $<60 \mathrm{ml} / \mathrm{min}$ ). CIN was defined as a post procedure rise in creatinine at day 3 of $>25 \%$ from baseline or an absolute rise of $44.5 \mu \mathrm{mol} / 1$. Severity of coronary disease was assessed using the SYNTAX Score and risk of CIN using the Mehran risk score. We evaluated serum and urine neutrophil gelatinase-associated lipocalin (NGAL) and albuminuria for additional information about CIN risk. $\mathrm{N}$-acetylcysteine and intravenous hydration were given to all patients with eGFR $<50 \mathrm{ml}$ in accordance with local guidelines. Results Baseline characteristics are summarised in table 1. 116 patients underwent coronary angiography and 92 underwent PCI. 39 patients $(18.8 \%)$ developed CIN. Contrast dose was similar in the CIN and non-CIN group $(\mathrm{p}=0.249)$. The Mehran risk score was strongly 
predictive of CIN development $(\mathrm{p}<0.001)$. The SYNTAX score did not differ between those who did or did not develop CIN ( $p=0.188$ ). A significant rise in serum NGAL was seen as early as $2 \mathrm{~h}$ post procedure in the CIN arm $(p=0.03)$ and this persisted at $4 \mathrm{~h}(\mathrm{p}=0.007)$ and $12-24 \mathrm{~h}(\mathrm{p}=0.0015)$. Urine NGAL levels did not change significantly during the first $24 \mathrm{~h}$. Neither albumin:creatinine ratio $(p=0.149)$ or protein:creatinine ratio $(\mathrm{p}=0.635)$ predicted development of CIN.

\section{Abstract 23 Table 1}

\begin{tabular}{lccc}
\hline & $\begin{array}{l}\text { No CIN outcome } \\
(\mathbf{n = 1 6 9 )}\end{array}$ & $\begin{array}{l}\text { CIN outcome } \\
(\mathbf{n = 3 9 )}\end{array}$ & p Value \\
\hline Age, (mean, SD) & $70.8(8.5)$ & $71.5(9.5)$ & 0.64 \\
Hypertension (\%) & $155(91.7)$ & $33(86.8)$ & 0.35 \\
Hyperlipidaemia (\%) & $165(97.6)$ & $37(97.4)$ & 0.92 \\
Previous MI (\%) & $66(39.1)$ & $15(39.5)$ & 0.96 \\
Ex or current smoker (\%) & $96(55.9)$ & $19(50.0)$ & 0.75 \\
Heart failure (\%) & $32(19.1)$ & $7(18.4)$ & 0.93 \\
Valvular heart disease (\%) & $28(16.6)$ & $7(18.4)$ & 0.78 \\
Family history IHD (\%) & $84(49.7)$ & $20(52.6)$ & 0.74 \\
BMI, (mean, SD) & $28.6(5.4)$ & $29.2(6.1)$ & 0.54 \\
\hline
\end{tabular}

Conclusions The current gold standard for measuring CIN is a rise in serum creatinine but this is of limited value as it does not increase until $48-72 \mathrm{~h}$ post renal injury. Neither the SYNTAX score, nor urinary albuminuria or proteinuria are predictive of CIN development. A rise in serum NGAL levels within the first $12 \mathrm{~h}$ following coronary angiography or PCI appears to be a very promising marker in the early diagnosis of CIN.

\section{PERCUTANEOUS MITRAL VALVE REPAIR WITH THE MITRACLIP DEVICE: A TERTIARY CARDIAC UK EXPERIENCE}

doi:10.1136/heartjnl-2011-300198.24

J Dungu, C S R Baker, M F Bellamy. Hammersmith Hospital, Imperial College London, London, UK

Introduction Percutaneous mitral valve repair using the transcatheter Mitraclip device is a novel therapy for patients with severe mitral regurgitation (MR) who are too high risk for conventional surgery. We report the largest UK series to date.

Methods Patients were screened with transthoracic (TTE) and transoesophageal echocardiography (TOE). Mitral regurgitation was graded by British Society of Echocardiography criteria. Twenty-four patients with $\geq$ grade $3+$ symptomatic MR underwent percutaneous mitral valve repair under general anaesthesia between February 2009 and October 2010. The Mitraclip device was deployed under 2- and 3-Dimensional TOE and fluoroscopic guidance. All patients were discussed with the manufacturing company (Evalve) and in a multidisciplinary meeting including $>2$ cardiologists and 2 cardiothoracic surgeons with a special interest in mitral valve surgery prior to being accepted.

Results Mitraclip therapy was attempted in 24 patients aged $71 \pm 11$ years with an average Euroscore of $16 \%$. The indication for intervention was functional MR in 10 patients (42\%), ischaemic MR in 7 patients (29\%) and degenerative MR in 7 patients (29\%). Twenty patients had successful deployment of the Mitraclip device (83\%). Fifteen patients $(75 \%)$ had 2 clips deployed. There were no vascular complications or strokes. We were unable to grasp the mitral valve leaflets in 2 patients due to an excessive coaptation gap. There was 1 procedural death due to leaflet tear in a patient with end-stage ischaemic cardiomyopathy and a grossly dilated left ventricle. All patients $(100 \%)$ treated with the Mitraclip had severe MR (grade 3 $+/ 4+$ ) prior to intervention. Mitral regurgitation was graded by colour Doppler alone following intervention as standard quantitative analyses are not validated in the presence of a Mitraclip. At 1-month follow-up to date, 10 patients (59\%) had a reduction in MR to $\leq$ grade $2+$ and 8 patients $(47 \%)$ had $\geq 2$ grade reduction in $M R$ $(p=0.001)$. The reduction in MR grade remained significant for the 8 patients with echo data at 6-month follow-up $(p=0.038)$. One patient had persistent grade $3+M R$ at 1-month follow-up due to late partial detachment of one of the 2 clips deployed. NYHA class reduced significantly following intervention. Prior to Mitraclip, 63\% of patients were in NYHA class III/IV. At 1-month follow-up postMitraclip only one patient (4\%) was in NYHA class III $(p=0.042)$ and 15 patients (83\%) had at least 1 grade reduction in NYHA class. There was no significant change in left ventricular size following intervention, although there was a trend towards reduced left ventricular volumes at 1-month follow-up (end diastolic volume 175 vs $160 \mathrm{ml}$, $\mathrm{p}=0.102$; end systolic volume 92 vs $79 \mathrm{ml}, \mathrm{p}=0.076$ ).

Conclusion In selected patients Mitraclip edge-to-edge repair successfully reduces the severity of mitral regurgitation and improves symptoms. Further studies are needed to examine whether these results are durable and associated with improved outcome.

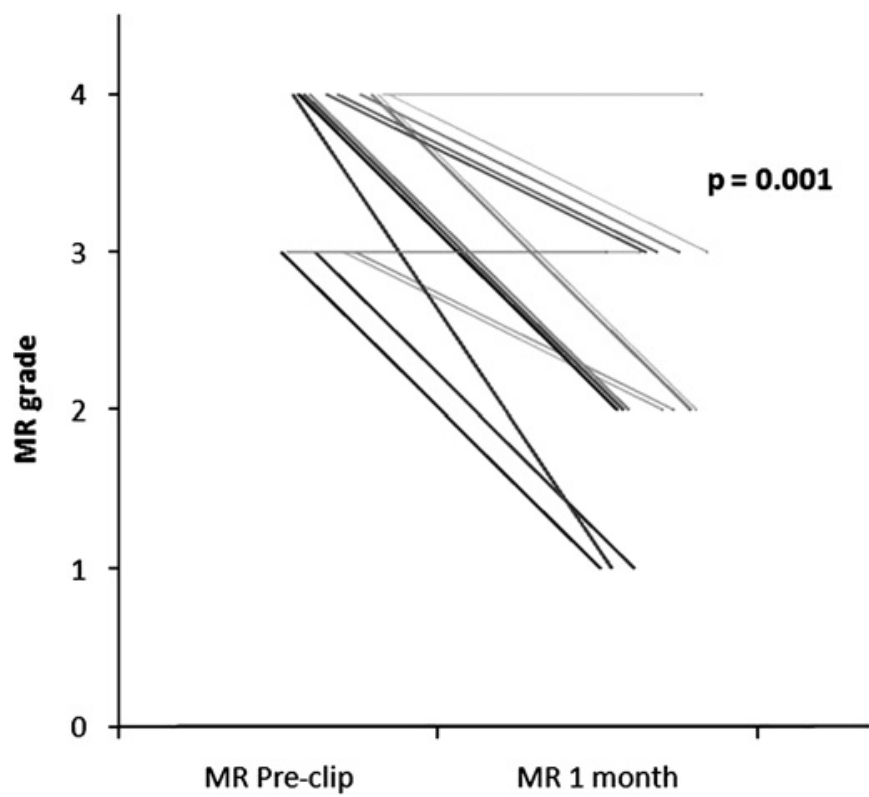

Abstract Figure 1 Change in MR grade post-Mitraclip.

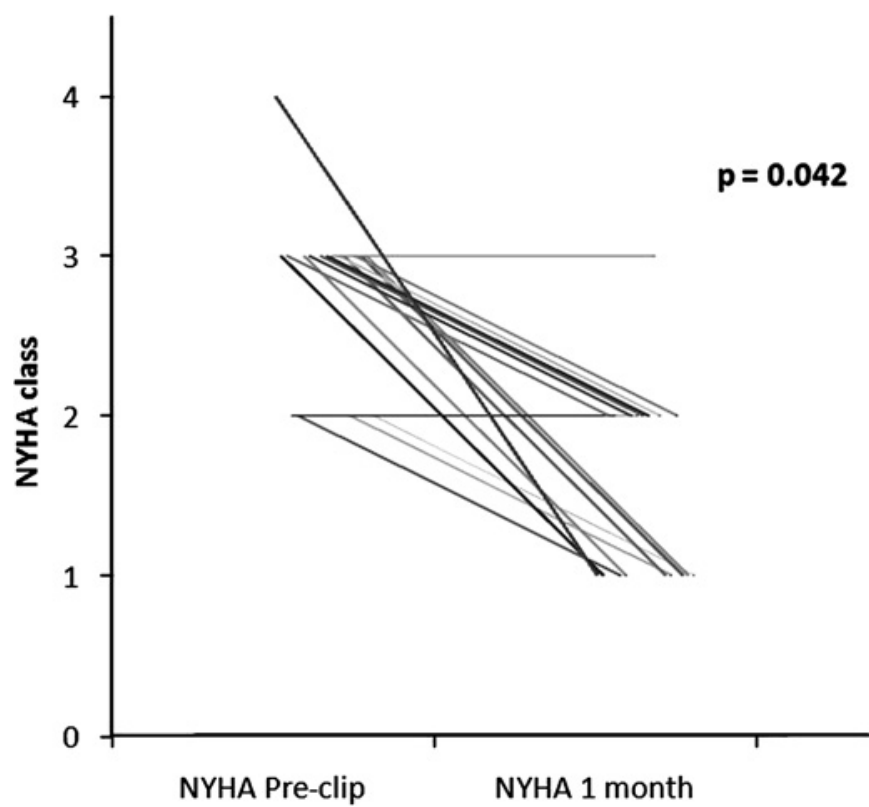

Abstract Figure 2 Change in NYHA class post-Mitraclip. 